\title{
IL CAREGIVER TRA TUTELA NORMATIVA, COMUNICAZIONE E STILE DI VITA
}

\author{
Giovanni Paruto ${ }^{1}$, Daniela Nuti Ignatiuk ${ }^{2}$ \\ ${ }^{1}$ Professore a contratto di Organizzazione dell'attività motoria e degli eventi sportivi nell'Università di \\ Bologna \\ ${ }^{2}$ Manager e organizzatrice di eventi
}

\begin{abstract}
Scopo di questo articolo è quello di far rilevare al lettore come quella che si definisce nel testo una categoria, cioè quella dei caregiver, è diventata in questi anni una categoria che il Legislatore deve tenere sempre più in considerazione al fine di migliorarne lo stile di vita. Il disegno di legge 1461 del 2019, ribadisce il valore sociale ed economico dell'attività di cura svolta da parte del caregiver familiare previsto già nella Legge 27 dicembre, n. 205 e introduce alcuni elementi quali: la nomina, i contributi figurativi e la tutela previdenziale, le agevolazioni nel settore sociale e le possibili detrazioni fiscali, la conciliazione tra assistenza e lavoro, le agevolazioni nel settore sociale e le possibili detrazioni fiscali e infine, l'accesso ai benefici, tutti elementi utili a caratterizzarne il ruolo. Considerando poi che il caregiver è sottoposto a un'attività fisica fatta di movimenti abituali e routinari, diventa determinante, per migliorarne le condizioni fisiche, l'uso di una apposita ed adeguata strumentazione che, rispetto all'età, aiuti a migliorarne le condizioni e lo stile di vita. L'articolo è un momento di riflessione per una categoria bisognosa di tutela normativa, una categoria che in silenzio aspetta un conforto, un riconoscimento ed una tutela dal Legislatore, una categoria soggetta a forte stress ossidativo, che ha bisogno di capire come rapportarsi e comunicare con l'ammalato o con il soggetto disabile che assiste, e che quando lo fa, riesce a farlo per esperienza e non attraverso appositi corsi formativi dei quali avrebbe estremo bisogno.
\end{abstract}

Keywords: Caregiver, Stress ossidativo, Comunicazione, Normativa

\begin{abstract}
The purpose of this article is to point out to the reader how what is defined in the text as a category, i.e. that of caregivers, has become in recent years a category that the legislator must take increasingly into consideration in order to improve their lifestyle. The draft law 1461 of 2019, reaffirms the social and economic value of the care activity carried out by the family caregiver already provided for in Law no. 205 of 27 December and introduces some elements such as: the appointment, notional contributions and social security protection, benefits in the social sector and possible tax deductions, conciliation between assistance and work, concessions in the social sector and possible tax deductions and finally, access to benefits, all useful elements to characterize their role. Considering then that the caregiver is subjected to a physical activity made up of habitual and routine movements, the use of a specific and adequate instrumentation that, in relation to age, helps to improve the conditions and lifestyle. The article is a moment of reflection for a category in need of regulatory protection, a category that silently awaits comfort, recognition and protection from the legislator, a category subject to strong oxidative stress, which needs to understand how to relate and communicate with the sick person or with the disabled person who cares, and that
\end{abstract}


when he does, he is able to do it through experience and not through special training courses which he would be in dire need of.

Keywords: Caregiver, Oxidative Stress, Communication, Regulation

\section{Stile di vita del caregiver e stress ossidativo}

Chi assiste un familiare svolge un lavoro estremamente importante per la società.

In Italia 7,3 milioni di persone assistono in famiglia, volontariamente, una persona cara non auto sufficiente. ${ }^{1}$ Solo nella Regione Emilia-Romagna nel 2019, ci sono stati oltre 220 mila caregivers, ${ }^{2}$ in maggioranza donne di età compresa tra 45 e 64 anni, che nel $60 \%$ dei casi hanno dovuto abbandonare la loro attività lavorativa. ${ }^{3}$

Si tratta di una categoria di lavoratori invisibili, senza alcuna tutela ma con latenti stress psico-fisici che per necessità seguono un familiare con gravi problemi di salute, molto spesso portatore di handicap.

È bene sottolineare che l'invalidità rappresenta la riduzione della capacità lavorativa, mentre l'handicap rappresenta la condizione di svantaggio sociale conseguente a una minorazione. Si tratta di due diverse condizioni: un soggetto con una invalidità al $100 \%$ può non essere portatore di handicap, mentre può esserlo una persona con una percentuale d'invalidità più bassa.

Tra tutti i tipi di assistenza esistono tassi di stress considerevoli e da non sottovalutare per l'organismo, quando l'assistenza riguarda un familiare (caregiver) che assiste un altro familiare. Solo per fare un esempio, possiamo considerare una forma di stress elevata rispetto alle altre, quella di coloro che assistono un familiare con demenza (caregiver di demenza). ${ }^{4}$ Questi soggetti, in genere donne con ruoli di mogli, figlie e in loro assenza badanti, purtroppo, rispetto ai caregivers che si occupano di familiari con altre patologie, hanno tassi più elevati di stress, depressione, ansia e tensione, ai quali si aggiungono molto spesso, anche problemi di salute in quanto lavorano spesso a tempo pieno. ${ }^{5}$ I caregivers dei pazienti con demenza sono la grande maggioranza. Sono in genere donne (74\%), di cui il $31 \%$ di età inferiore a 45 anni, il 38\% di età compresa tra 46 e 60, il 18\% tra 61 e 70 e ben il 13\% oltre i 70 anni $^{6}$.

Ma questo è solo un esempio, in quanto lo stress che i caregivers assumono con la loro attività assistenziale coinvolge tutta la categoria, qualsiasi problema di salute abbia il loro familiare.

Vi sono diversi studi nella letteratura medico scientifica che indicano come lo stress ossidativo rivesta un ruolo determinante sia nell'invecchiamento precoce che in alcune gravi patologie alle quali il caregiver è esposto ${ }^{7}$.

Le cause che possono portare ad un'alterazione dell'equilibrio del nostro organismo, sono molte e tra queste una delle più diffuse è quella legata allo stile di vita.

Quando si parla di stile di vita si intende un insieme di abitudini che caratterizzano il modo di vivere di una persona. Nel definire lo stile di vita, andiamo a comprendere tutte le abitudini alimentari e comportamentali, tra cui va presa in seria considerazione anche la capacità di gestire lo stress. Lo stress è una condizione esterna al nostro organismo che contribuisce notevolmente a generare uno squilibrio poiché scatena molti meccanismi

\footnotetext{
${ }^{1}$ Caregiver familiare: chi è, quanti sono e cosa prevede la legge, in www.osservatoriodiritti.it/caregiver-familiare, $1^{\circ}$ ottobre 2019.

${ }^{2}$ Si parla di caregiving familiare quando chi presta assistenza è un parente del malato, ad esempio il marito, il fratello oppure il figlio o la figlia e di caregiving professionale, dove chi presta cure è personale specializzato e abilitato, es. infermiere, badante, assistente domiciliare, OSS, ecc. Le cure che i caregivers prestano sono sia di tipo fisico (pulizia e igiene, pasti, medicazioni), sia di tipo pratico (organizzare visite, riabilitazioni, somministrazione medicine, ecc.), sia di tipo emotivo (supportando il proprio assistito, stimolandolo con domande e conversazioni, intrattenendolo con giochi, film, letture, ecc.).

${ }^{3}$ Linee attuative della legge regionale n. 2 del 28 marzo 2014. Atti amministrativi della Giunta Regionale, delibera n. 858 del 16 giugno 2017.

${ }^{4}$ Studi effettuati in 31 Paesi europei hanno stimato che i pazienti affetti da demenza sono 7,3 milioni (Alzheimer Europe su dati Euro Code, 2009). In Italia vi sono circa 1 milione di pazienti colpiti e circa 3 milioni di persone sono coinvolte direttamente o indirettamente nell'assistenza come caregiver (Euro Code, 2009).

${ }^{5}$ A. Bokov et al., The role of oxidative damage and stress in aging, in Mechanisms of ageing and development, 2004, 10-11, p. 125.

${ }^{6}$ Caregiver e demenza: la salute di chi cura, in www.igeacps.it.

${ }^{7}$ K.C. Kregel, H.J. Zhang, An integrated view of oxidative stress in aging: basic mechanisms, functional effects, and pathological considerations, in American journal of physiology. Regulatory, integrative and comparative physiology, 2007, 1, p. 292.
} 
biologici atti a produrre energia per il corpo. Questi processi sono responsabili della generazione di una elevata quantità di radicali liberi. Lo stress ossidativo è una condizione inevitabile perché è parte delle normali funzioni biologiche dell'organismo. L'aumento di richiesta di energia dovuta allo stress è una conseguenza della normale risposta del sistema nervoso autonomo (SNA) ${ }^{8}$ a tale condizione.

Il sistema nervoso autonomo ha il compito di:

- regolare in maniera indipendente dalla nostra volontà, il funzionamento di organi e tessuti (es. l'attività cardiaca), regolandoli a seconda delle necessità;

- reagire a stimoli esterni (esogeni) come lo stress o condizioni di pericolo, regolando il corpo per affrontarli e garantire la nostra sopravvivenza;

- reagire anche a stimoli interni (endogeni) che hanno lo scopo di ricondurre il funzionamento di organi, tessuti e muscoli a funzioni rigenerative, come la digestione e la rigenerazione delle cellule.

Durante i periodi particolarmente stressanti nel corpo del caregiver prevale la risposta a stimoli di stress. Questo induce 1'organismo ad organizzarsi per affrontare una situazione di potenziale pericolo e ad attivare sé stesso esattamente come se dovesse essere a rischio l'esistenza stessa (percezione del sistema nervoso autonomo). In questi momenti di stress i muscoli e gli organi si preparano a gestire una fuga o un attacco per difendersi. Il nostro corpo non distingue se lo stress è realmente un pericolo oppure no, semplicemente si prepara ad affrontare un potenziale rischio con l'obbiettivo di difendere la propria esistenza.

Questo meccanismo attiva nel caregiver una serie di processi biologici che hanno lo scopo di fornire la necessaria energia e gli elementi per affrontare una condizione così critica. Il caregiver, nelle situazioni di forte stress derivanti dall'attività di assistenza di una persona, deve mettere in campo una grande energia, sia volontariamente per affrontare la molteplicità di situazioni che si trova a gestire, sia involontariamente per una richiesta dell'organismo che deve sopportare uno stress particolarmente elevato che ha, quali conseguenze, un significativo aumento nell'organismo della produzione di radicali liberi.

In queste condizioni si crea una sorta di spirale dove il caregiver, per supportare lo stress, aumenta la produzione di radicali liberi che danneggiano il corpo e riducono la capacità di ripristinarne il corretto equilibrio. Questo effetto porta ad aumentare le possibilità di innescare patologie in cui lo stress ossidativo è parte attiva nell'attivazione e nel suo decorso.

La figura del caregiver è definita "categoria", non solo per il lavoro che questi soggetti svolgono, ma in quanto soggetti sottoposti ad un elevato stress e quindi, a squilibri non voluti del proprio corpo, come appunto lo stress ossidativo.

Il termine "stress ossidativo", entrato negli ultimi anni nel lessico quotidiano, indica l'insieme delle alterazioni che si producono nei tessuti, nelle cellule e nelle macromolecole biologiche, allorché queste rimangono esposte ad un eccesso di agenti ossidanti.

Quando si parla di uno stato di stress ossidativo elevato, si parla di una condizione riconosciuta come negativa per il nostro corpo ed il caregiver, rispetto ad altri soggetti meno coinvolti in situazioni simili, pur se non esenti da stress, ne è molto più interessato.

In tutti gli organismi che vivono in ambiente aereo, vi è un equilibrio tra la produzione di sostanze ossidanti e antiossidanti. Questo equilibrio è essenziale per tutta una serie di funzioni fisiologiche del corpo, le quali sono sensibili ad una valutazione di questo delicato equilibrio.

Queste alterazioni possono provocare effetti che danneggiano le componenti della cellula incluse le proteine, $\mathrm{i}$ lipidi e gli acidi nucleici.

I radicali liberi vengono continuamente prodotti dal nostro organismo attraverso numerosi processi biochimici. Una determinata quantità di sostanze ossidanti è infatti indispensabile per mantenere il corretto funzionamento delle cellule, regolando l'equilibrio del nostro corpo. Durante le normali funzioni biologiche, i radicali liberi prodotti possono superare il valore di soglia rispetto a quelli necessari. Tale valore aumenta ulteriormente quando

\footnotetext{
${ }^{8}$ Il sistema nervoso autonomo è quella zona del sistema nervoso periferico che, oltre ad alcuni muscoli, controlla le funzionalità degli organi interni quali il cuore, lo stomaco e l'intestino. Il sistema nervoso autonomo può essere così suddiviso: sistema nervoso simpatico, parasimpatico e metasimpatico.
} 
una persona vive condizioni altamente stressanti, come ad esempio il caregiver. Se questa quantità eccedente di radicali liberi non viene neutralizzata dai meccanismi naturali di riequilibrio del nostro corpo, si possono instaurare danni all'interno della cellula, in grado di ridurre le funzioni della stessa fino a farla cessare completamente.

Le cause che generano questo squilibrio possono provenire dal nostro stesso corpo tramite le normali funzioni o a causa dell'esistenza di problematiche anche non gravi, ma soprattutto a causa di uno stile di vita non ottimale o da condizioni ambientali.

Gli effetti dello stress ossidativo sull'organismo sono in costante osservazione e la lista delle patologie che vi si possono ricondurre è in costante aumento. L'effetto dello stress ossidativo sul corpo è un elemento che per la categoria dei caregiver e non solo, va considerato come una delle cause di origine di diverse patologie. Per questo motivo è importante cercare di mantenere un buon equilibrio tra i radicali liberi prodotti e la naturale capacità di contrastare il loro effetto da parte del nostro organismo.

Lo stress ossidativo è, quindi, parte del processo che porta a sviluppare una patologia, ma è anche parte del decorso della patologia stessa. È un elemento che deve essere tenuto in grande considerazione al fine di mantenere un buon livello di salute. Infatti, benché non lo si possa considerare come l'origine di tutti i processi patologici, rimane comunque un fattore determinante.

\section{La possibile normativa di riferimento}

Rispetto ad altri paesi l'Italia risultava uno dei pochi paesi in Europa dove non era stata riconosciuta e dove non è, ad oggi, tutelata anche da un punto di vista previdenziale, la figura del caregiver familiare.

Il Legislatore ha riconosciuto questo ruolo di assistenza ed è intervenuto, nel 2017, con la Legge di bilancio $2018,{ }^{9}$ per definire alcuni aspetti di quello che possiamo considerare un lavoro. Così è stata data una definizione e un riconoscimento alla figura, cercando di colmare quel vuoto normativo in modo da allineare il nostro Paese alle tutele che altri paesi europei avevano previsto già da tempo.

L'articolo 255 della citata legge 205/2017, definisce il caregiver familiare come la persona che assiste e che si prende cura del coniuge, dell'altra parte dell'unione civile tra persone dello stesso sesso o del convivente di fatto, di un familiare o di un affine entro il secondo grado, ovvero, nei soli casi indicati dall'articolo 33, comma 3, della legge 5 febbraio 1992, n. 104, di un familiare entro il terzo grado ${ }^{10}$ che, a causa di malattia, infermità o disabilità, anche croniche o degenerative, non sia autosufficiente e in grado di prendersi cura di sé, sia riconosciuto invalido in quanto bisognoso di assistenza globale e continua di lunga durata ai sensi dell'articolo 3, comma 3, della legge 5 febbraio 1992, n. 104, o sia titolare di indennità di accompagnamento ai sensi della legge 11 febbraio 1980, n. 18.

Per definire la materia il 7 agosto 2019 è stato presentato in Senato il disegno di legge n. 1461 "Disposizioni per il riconoscimento ed il sostegno del caregiver familiare". Il testo si compone di 11 articoli ed è attualmente in discussione presso la $11^{\mathrm{a}}$ Commissione permanente del Senato (Lavoro pubblico e privato, previdenza sociale).

Descrivere i contenuti della proposta di legge aiuta a comprendere come finalmente il Legislatore ha apprezzato l'importanza del ruolo del caregiver familiare nella società civile. Il disegno di legge n. 1461 ribadisce il valore sociale ed economico dell'attività di cura svolta da parte del caregiver familiare previsto già nella Legge 27 dicembre, n. 205 e introduce alcuni elementi, quali la nomina, i contributi figurativi e la tutela previdenziale.

\section{La nomina}

È l'assistito quando ancora cosciente a nominare il caregiver. Lo fa personalmente $o$ attraverso l'amministratore di sostegno, ovvero, nei casi di interdizione o di inabilitazione, attraverso il tutore o il curatore. Tale nomina può essere revocata, da parte dell'assistito, in qualsiasi momento. L'atto di nomina del caregiver

\footnotetext{
${ }^{9}$ Legge 27 dicembre 2017, n. 205, art. 255.

${ }^{10}$ Bisnonno o bisnonna pronipote (figlia o figlio del nipote); nipote (figlia o figlio del fratello o della sorella); zio e zia (fratello o sorella del padre o della madre).
} 
familiare, sottoscritta dallo stesso assistito può avvenire anche attraverso videoregistrazione o mediante un qualsiasi altro dispositivo.

La nomina, che può essere assunta da un solo familiare dell'assistito, cessa dallo stato giuridico e dalla funzione nei casi di decesso dell'assistito o qualora l'assistito non abbia più i riconoscimenti richiesti di stato di gravità o invalidità (in quest'ultimo caso, salvo i casi in cui sia affetto da patologie oncologiche).

\section{I contributi figurativi e la tutela previdenziale}

Il disegno di legge prevede che al caregiver familiare (non lavoratore) vengano riconosciuti, fino ad un massimo di tre anni, i contributi figurativi ${ }^{11}$ (utili sia per il conseguimento del diritto alla pensione sia per il suo calcolo), equiparati a quelli del lavoro domestico (collaboratore familiare). Tali contributi potranno aggiungersi a quelli eventualmente già versati precedentemente dal caregiver per altre attività lavorative. Dopo l'approvazione del disegno di legge bisognerà aspettare le varie circolari dell'INPS che dovranno integrare la figura del caregiver tra i fruitori di questi contributi.

Il riconoscimento dei contributi figurativi a carico dello Stato avviene previa dichiarazione delle ore di assistenza da presentare all'INPS ogni trimestre. Da notare che l'assistenza del caregiver, ad esempio una mamma quando assiste un figlio disabile dalla nascita o dalla prima infanzia, fa apparire poco aderente alla norma sia la dichiarazione delle ore, a causa della continuità della sua assistenza, sia i tre anni di contributi figurativi previsti dal disegno di legge, che risultano davvero molto pochi, così come poche sono le risorse finanziarie poste a loro copertura.

Ai caregivers la CONFAD (Coordinamento Nazionale Famiglie con Disabilità), chiede che venga riconosciuto il diritto di accedere al prepensionamento anticipato e senza penalizzazioni, proporzionalmente agli anni di cura prestati al familiare, al raggiungimento dei 35 anni di contributi, sommando ai contributi del lavoro principale quelli figurativi versati dallo Stato per l'attività di caregiver familiare, sia nel caso che il cargiver abbia svolto in precedenza o stia ancora svolgendo un'attività lavorativa, sia nel caso in cui lo stesso non abbia mai potuto lavorare. All'entrata in vigore della legge, il calcolo dei contributi figurativi dovrà essere retroattivo per i caregivers che prestano ancora l'attività di cura al proprio familiare.

\section{Le iniziative degli Enti locali territoriali}

La speranza è che la maggioranza al Governo e i partiti tutti, si attivino allo scopo di ottenere un congruo aumento della dotazione finanziaria prevista, pari a 25 milioni di euro per ogni anno del triennio 2019/2021. ${ }^{12} \mathrm{La}$ legge di bilancio del 2018 aveva previsto 20 milioni di euro per ciascuno degli anni 2018, 2019 e 2020, ma il decreto ministeriale di attuazione (che avrebbe dovuto essere emanato entro il 31 marzo 2018) non è mai stato predisposto. Il decreto avrebbe dovuto contenere la disciplina delle tipologie di aiuti ammissibili, i criteri di riparto del fondo tra le Regioni, i requisiti e i criteri di accesso. Alcune Regioni e alcuni Comuni, nel frattempo, dando rilevanza al lavoro sociale che il caregiver svolge, sono intervenuti con norme di settore, ribadendo e definendo la figura così come ben specificata nella Legge 205 del 2017, ma con modalità di intervento e fondi economici da destinare, anticipando l'intervento statale.

\section{La Regione Emilia-Romagna}

Per sostenere e tutelare questa forma di assistenza volontaria la Regione Emilia-Romagna, prima in Italia, nel

${ }^{11}$ I contributi figurativi sono contributi accreditati senza oneri a carico del lavoratore per periodi in cui l'interessato è costretto a interrompere l'attività lavorativa per motivi diversi, quali la gravidanza, la malattia, la disoccupazione.

${ }^{12}$ La legge di bilancio del 2018 aveva previsto una dotazione iniziale di 20 milioni di euro per ciascuno degli anni 2018, 2019 e 2020, destinato alla copertura finanziaria di interventi legislativi finalizzati al riconoscimento del valore sociale ed economico dell'attività di cura non professionale del caregiver familiare. Per un approfondimento si rimanda alla lettura degli Atti parlamentari della discussione sulla legge avvenuta in Senato, n. 46, XVIII Legislatura. Mentre, con il comma 483 dell'articolo 1 della legge n. 145 del 30 dicembre 2018 (legge di bilancio 2019), il Fondo in parola è stato incrementato di 5 milioni di euro per ciascuno degli anni 2019,2020 e 2021. Pertanto, la rimodulazione complessiva della dotazione del Fondo è di 25 milioni negli anni 2019, 2020 e 2021. 
2014 si è dotata di una legge specifica che ha consentito alle aziende sanitarie e ai servizi socio-sanitari in capo ai Comuni, di realizzare vari interventi. La Regione, sull'esempio di altri Paesi europei è stata la prima regione in Italia ad avere adottato una legge che riconosce il ruolo del familiare, del convivente, della persona amica, che si prende cura di una persona cara impossibilitata a farlo autonomamente. Una scelta seguita poi da altre regioni, come Lombardia, Abruzzo, Campania, Lazio, Marche Piemonte, Puglia e Sardegna, ed altre che si stanno attivando per adottare provvedimenti simili. Con le risorse del fondo regionale per la non autosufficienza (si stima che in Emilia-Romagna, le persone che si prendono cura dei propri cari non autonomi siano oggi - maggio 2020 circa 250 mila, cioè il $5 \%$ della popolazione residente), la Regione sostiene una serie di interventi diretti e indiretti a favore dei caregivers e dei loro famigliari non autosufficienti. Gli interventi più significativi riguardano l'assegno di cura (sostegno economico a favore delle famiglie che assistono a casa un anziano o disabile grave) concesso, nel solo 2016, a 9.000 anziani, 2.100 disabili gravi e gravissimi, e contributi aggiuntivi per sostenere i costi delle assistenti familiari (badanti) a 4.200 famiglie. La Regione inoltre prevede la possibilità da parte delle famiglie di utilizzare alcuni servizi di assistenza per alcune ore del giorno o brevi periodi dei propri congiunti. Rientrano tra questi l'accoglienza temporanea di sollievo in strutture residenziali, ${ }^{13}$ fruita a livello regionale da circa 2.900 persone; i caffè Alzheimer e i Meeting center (centri di accoglienza diurna per anziani affetti da tale patologia) frequentati da 3.400 persone. A questi interventi si aggiungono una serie di iniziative specifiche per i caregivers. Si va da quelle formative e di qualificazione del lavoro di cura (1500 partecipanti) a quelle di consulenza e sostegno per l'adattamento dell'ambiente domestico (1.725 operatori, familiari, caregivers e volontari). Infine, per i giovani caregivers che si prendono cura regolarmente di un familiare, sacrificando tempo ed energie per la famiglia e rischiando di pagare un prezzo alto in termini di salute e realizzazione personale, sono previsti accordi quadro con istituzioni scolastiche e universitarie per garantire loro supporto e agevolazioni nello svolgimento del percorso di studio. ${ }^{14}$

Secondo l'ultima indagine multiscopo condotta dall'Istat, nel 2011 in Emilia-Romagna la maggioranza dei caregivers familiari è donna ed è compresa tra i 45 e i 64 anni; una buona parte è impegnata ad assistere più di una persona (nella combinazione bambini e anziani). In Emilia-Romagna, il 12,5\% delle donne (10,7\% in Italia) e 1' $\%$ degli uomini (6,2\% in Italia) di età compresa tra i 15 e i 64 anni si dichiarano caregivers di adulti disabili. I "giovani caregivers", cioè quelli di età compresa tra i 15 ed i 24 anni, in regione si stima siano 13.250 (169.000 in Italia). ${ }^{15}$

Secondo un'indagine ISTAT del 2015, in Italia sarebbero addirittura 8,5 milioni i caregivers, di cui 7,3 milioni caregivers familiari. Di questi sono 2.146 .000 coloro i quali dichiarano un impegno assistenziale superiore alle 20 ore settimanali. ${ }^{16}$

La stessa Istat, in una indagine multiscopo del 2010, aveva stimato in 3.329.000 le persone che, nel contesto familiare, si prendono cura regolarmente di anziani, di malati e di persone disabili, senza però in quella occasione dare indicazioni sull'entità dell'impegno assistenziale. Si tratta prevalentemente di donne (74\%), di cui il $31 \%$ di età inferiore a 45 anni, il $38 \%$ di età compresa tra 46 e 60 , il $18 \%$ tra 61 e 70 e ben il $13 \%$ oltre i $70 .{ }^{17}$

\section{Il Comune di Bologna}

Tra i Comuni, quello di Bologna ha stanziato nel 2020 un milione di euro in servizi rivolti ai caregivers. L'obiettivo è stato quello di fornire servizi, assistenza, supporto e manutenzione domestica, dando risposte

\footnotetext{
${ }^{13}$ Nelle Case-residenze per anziani non autosufficienti (Case protette/RSA) possono essere previsti dei posti letto utilizzabili per ricoveri temporanei, che possono rappresentare dei veri e propri ricoveri di sollievo per garantire un periodo di riposo alle famiglie che scelgono di assistere gli anziani a casa e di non ricorrere al ricovero definitivo, oppure che improvvisamente non possono provvedere all'assistenza per motivi di salute o altri motivi contingenti. Può essere prevista una quota di compartecipazione (retta) ridotta a favore degli utenti grazie ad un maggiore contributo a carico del Fondo regionale per la non autosufficienza (delibere Giunta Regionale $n$. 273/2016 e n. 1206/07).

${ }^{14}$ Caregiver, Bonaccini: $7 \mathrm{mln}$ per sostenere chi fa assistenza, in www.regioni.it, 13 gennaio 2020.

${ }^{15}$ I dati sono estratti da www.confcooperativemiliaromagna.it.

${ }^{16}$ Rapporto Istat Conoscere il mondo della disabilità: persone, relazioni e istituzioni, in www.istat.it, 12 dicembre 2018.

${ }^{17}$ Caregiver in Italia, Chi sono, quanti sono, cosa fanno, in www.lenius.it.
} 
tempestive a quelle problematiche che uniscono i servizi sociali e socio-sanitari.

Il Comune è intervenuto in merito ai servizi, sulla gestione delle pratiche e degli adempimenti da svolgere: con supporto e consulenza tesi ad approfondire esigenze specifiche e ad orientare il caregiver sulle opportunità che il sistema sia pubblico che privato può offrire a suo vantaggio o a vantaggio dell'assistito (pratiche INPS, richiesta dell'invalidità civile o del riconoscimento di 1. 104/92; SPID; ISEE; domiciliazione delle utenze; spesa domicilio, ritiro ricette mediche farmaci, prenotazione visite mediche e accertamenti diagnostici). Infine, il Comune ha previsto il suo intervento mediante gli accompagnamenti con automezzo comunale o pubblico (per visite mediche da realizzarsi anche in sostituzione del caregiver, visite per invalidità civile, uffici postali, banca, attività di socializzazione, visite cimitero, ecc.), da svolgersi anche mediante convenzioni con Associazioni di volontariato. ${ }^{18}$

\section{Le agevolazioni nel settore sociale e le possibili detrazioni fiscali}

$\mathrm{Al}$ fine di sostenere le attività e la figura del caregiver familiare, il disegno di legge 1461 del 2019, definisce i Livelli Essenziali delle Prestazioni (LEP) da garantire al caregiver nel campo sociale, tra cui:

- servizi ed interventi di sollievo ed emergenza o programmati, erogati attraverso enti territoriali, ASL, mediante operatori socio-sanitari o socio-assistenziali;

- consulenze per l'adattamento domestico

- formazione e informazione sulle competenze;

- $\quad$ supporto psicologico; percorsi preferenziali nelle strutture sanitarie per ridurre i tempi di attesa sia per l'assistito che per il caregiver;

- rilascio di tessera di riconoscimento per avere priorità nel disbrigo di pratiche amministrative.

Con lo stesso obiettivo, il disegno di legge si propone di inserire nei Livelli Essenziali di Assistenza (LEA) nuovi servizi e prestazioni appositamente per i caregivers e i loro assistiti. In particolare, la domiciliazione di visite e prestazioni specialistiche, che permetterebbe così al caregiver e al familiare di sottoporsi a visite ed esami nel proprio domicilio. Ben venga la domiciliazione delle visite, ma la stessa domiciliazione avrebbe un senso maggiore se fosse inserita in un contesto più ampio di vigilanza sanitaria che richiederebbe un riconoscimento delle patologie professionalmente acquisite.

La proposta di legge citata, inoltre, come forma di maggior tutela, introduce detrazioni fiscali del $50 \%$, riservate ai caregivers familiari, per attività di cura e assistenza su una spesa massima di 10.000 euro annui.

\section{La conciliazione tra assistenza e lavoro}

Per i caregivers familiari e lavoratori, si prevedono degli interventi per favorire la conciliazione tra le attività lavorative e quelle di assistenza. Il lavoro svolto dal caregiver rappresenta per il Paese un valore sociale ed economico, per questo e a maggior ragione, il caregiver lavoratore deve poter usufruire di tutti i benefici e di tutte le possibili tutele. La norma in esame prevede per il caregiver lavoratore:

- il diritto di rimodulare l'orario di lavoro compatibilmente con l'attività di assistenza;

- il diritto prioritario di scelta della sede di lavoro tra quelle disponibili più vicine alla casa dell'assistito. i.

\section{Il riconoscimento delle competenze del caregiver}

L'articolo 8 del disegno di legge 1461 prevede che l'esperienza maturata in qualità di caregiver possa essere riconosciuta, anche ai fini di un successivo accesso o reinserimento lavorativo, con una formalizzazione e certificazione o come credito formativo per l'acquisizione della qualifica di operatore socio-sanitario o di altre figure professionali dell'area socio-sanitaria. Questo articolo appare molto generico e non definisce chiaramente chi deve riconoscere le competenze o il valore del credito formativo rapportato al tempo di assistenza.

L'attenzione del Legislatore dovrebbe essere quella di garantire, attraverso corsi formativi, livelli di preparazione per coloro che assistono i familiari e che sono impegnati nell'assistenza domiciliare. Lo scopo è quello di avvicinare la loro preparazione a quella di coloro che assistono i malati presso le strutture di ricovero,

\footnotetext{
${ }^{18} \mathrm{Al}$ via il progetto di supporto ai caregivers, in www.comune.bologna.it, 10 febbraio 2020.
} 
sino a pensare di programmare corsi omogenei di preparazione. Ipotesi questa molto affascinante, ma poco realizzabile, considerando che ci troviamo spesso difronte a donne e uomini di una certa età e a casi minori di caregivers giovani tra i quali molti fratelli.

In assenza di interventi legislativi idonei, la preoccupazione è che l'assistenza domiciliare potrebbe determinare la formazione di una nuova e ampia area di lavoro sottopagato. La figura del caregiver familiare dovrebbe essere valorizzata e sostenuta dallo Stato e le idee di tutela dovrebbero avere come riferimento quanto rilevato dallo studio di Elizabeth Helen Blackburn, premio Nobel per la medicina nel 2009, che nelle sue ricerche ha rilevato come l'aspettativa di vita per i caregivers familiari possa ridursi anche di 17 anni e questo tutti noi vorremmo che non accadesse.

Rimane il fatto che le famiglie hanno bisogno di aiuti sociali ed emotivi.

Basti per esempio pensare alla scoperta di un figlio affetto da autismo. In questi casi, la famiglia dovrebbe essere aiutata a mantenere il tipo di vita e le relazioni sociali precedenti alla nascita del figlio autistico con la garanzia da parte delle istituzioni che ci sia un intervento di personale competente in grado di consentire le relazioni sociali, nonché, se la madre è una lavoratrice, di mantenere il posto di lavoro secondo quanto previsto dal disegno di legge in discussione. Gli aiuti sociali ed emotivi dovrebbero essere estesi anche agli eventuali fratelli del bambino e sarebbe auspicabile che un'attenzione particolare venisse rivolta anche ai nonni. Ad esempio, per aiutare i familiari si potrebbero ipotizzare possibili brevi periodi di vacanza per un giusto riposo per potersi "ricaricare" e trovare nuove energie.

\section{L'accesso ai benefici}

Per accedere ai benefici il caregiver familiare è tenuto a presentare all'Istituto nazionale della previdenza sociale (INPS) i seguenti documenti:

a) atto di nomina di caregiver familiare, sottoscritto dall'assistito o tramite registrazione o altro;

b) estremi del verbale di riconoscimento dello stato di gravità dell'assistito ai sensi dell'articolo 3, comma 3, della legge n. 104 del 1992, ovvero del verbale di riconoscimento dell'invalidità del medesimo ai sensi della legge n. 18 del 1980;

c) autocertificazione di residenza in un comune del territorio italiano. Per i cittadini extracomunitari, l'autocertificazione di residenza in un comune del territorio italiano per almeno dieci anni, gli ultimi due dei quali in modo continuativo;

d) per i cittadini extracomunitari, copia del permesso di soggiorno UE per soggiornanti di lungo periodo. ${ }^{19}$

\section{La delicatezza della comunicazione}

Venendo ora ad un altro aspetto fondamentale della figura del caregiver è necessario considerare che quando in una famiglia un parente si ammala di Parkinson, di Sla o di Alzheimer, quando a causa di un incidente non è più autosufficiente, quando non lo è dalla nascita o quando la malattia appare nei primi anni di vita, la comunicazione diventa particolarmente delicata.

Il ruolo del caregiver è fondamentale per tanti aspetti, in particolare, se la malattia diventa cronica e quando la sua funzione diventa quella di rivestire un ruolo nuovo e diverso rispetto al passato. Egli riveste un ruolo importantissimo nell'ambito delle cure ai pazienti con malattie croniche in quanto, oltre all'assistenza vera e propria, deve occuparsi degli aspetti più pratici dell'assistenza, come ad esempio, accompagnare in ospedale il proprio parente, contattare i medici, acquistare i farmaci necessari, informarsi sui possibili ed eventuali effetti collaterali delle medicine e delle cure e seguire tutto quello che riguarda le pratiche burocratiche da espletare, quali l'iter per l'invalidità del familiare, la dichiarazione dei redditi, le pratiche per eventuali ricoveri, ecc.

Senza comunicazione non esiste relazione e senza relazione non si struttura quel rapporto di cooperazione necessario per stabilire una alleanza terapeutica fatta di aiuti nelle situazioni di difficoltà dell'ammalato e di mantenimento delle sue funzioni, nel rapporto tra caregiver e ammalato. La comunicazione efficace consente di

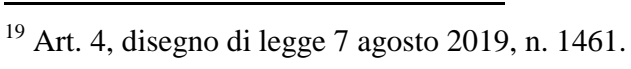


esprimersi verbalmente e non, in modo congruo alla propria cultura, sia in relazione al tipo di rapporto in essere (caregiver/familiare ammalato o disabile) sia quando si è in grado di ascoltare in modo attento ed empatico.

All'esterno del rapporto familiare, cioè nel rapporto con il medico o con lo psicologo, l'alleanza terapeutica, in particolare in presenza di bambini autistici, comporta le seguenti considerazioni:

- il medico dovrebbe essere consapevole e rispettoso della storia familiare, delle abitudini, dei valori sociali, culturali e religiosi;

- dovrebbe comprendere i problemi dei genitori i quali spesso si sentono colpevoli della malattia del proprio figlio.

Il medico diventa una guida che deve capire ed integrare il proprio operato con quello dei genitori. Egli dovrebbe considerare i bisogni globali della famiglia, tenendo conto delle esigenze degli altri figli e della situazione lavorativa e sociale dei genitori. ${ }^{20}$

Certamente i modi e i livelli della comunicazione dipendono dalle capacità cognitive del familiare ammalato e da quelle del familiare che lo assiste, cioè il caregiver, pertanto è importante che chi assiste, riesca a rapportarsi nei modi più adeguati alla situazione già dal momento traumatico in cui gli viene comunicata la malattia del familiare.

\section{Il livello della comunicazione}

Il livello della comunicazione è verbale, para verbale e non verbale, senza mai dimenticare che una modalità fondamentale della comunicazione è l'ascolto, in particolare l'ascolto empatico.

La comunicazione verbale è costituita dalle parole che usiamo quando parliamo o scriviamo, e normalmente è il livello di comunicazione che curiamo con maggiore attenzione. Il soggetto che assiste un familiare la cui capacità di comunicazione è compromessa, deve comunicare nella maniera più semplice possibile. Questo modo di comunicare deve tener conto di un linguaggio semplice; formulando parole chiare dette lentamente; non ponendo mai più di una domanda alla volta; parlando con voce calma e con un modo di agire rassicurante nei confronti del soggetto assistito; mostrando approvazione per tutto quello che l'assistito riesce a fare senza il suo aiuto, sorridendogli con un'espressione di affetto.

Fondamentale per il caregiver è saper dire di no senza sentirsi in colpa. Non è piacevole per nessuno dire di "no", in particolare quando il no viene pronunciato nei confronti di un parente che assistiamo, ma diventa essenziale quando non sono presenti elementi obiettivi per dire di "sì" e quando dire di "sì" non aiuta né noi, né la persona che assistiamo. Certamente il nostro "no" va motivato, spiegato, espresso in modo assolutamente non aggressivo e suggerendo, con delicatezza, eventuali alternative.

Il livello para verbale riguarda il tono della voce, il ritmo della voce, il volume della voce, l'espressività della voce, le pause, i silenzi, i sospiri. L'insieme, insomma, delle caratteristiche della voce e del modo di pronunciare il messaggio.

Il livello non verbale consistente nella postura, nella gestualità, nella mimica (espressioni del volto, sguardo, sorriso), nei movimenti delle mani, negli odori, nell'abbigliamento e nell'aspetto esteriore,

L'importanza di questi livelli di comunicazione è enorme: il livello para verbale e quello non verbale permettono infatti al caregiver di toccare, a livello comunicativo, realmente il parente malato o disabile. La comunicazione non verbale assolve diverse funzioni in quanto consente al caregiver di esprimersi completamente, di sentirsi più in stretto contatto e di ricevere dal proprio caro, ammalato o disabile, le sue emozioni: per entrambi un volto teso, una carezza, un sorriso, la voce, quando si incrina per l'emozione, indicano un disagio che stanno vivendo.

Comunicare bene con la persona ammalata o disabile vuol dire osservare piccoli dettagli comportamentali che non avremmo mai previsto e mai immaginato, vuol dire adeguarsi ad un altro modo di vedere le situazioni, ad esempio: la gestualità deve essere spontanea, il tono della voce deve essere adeguato e con toni variabili, mentre il linguaggio deve essere semplice, naturale, incisivo ma dolce e sintetico.

\footnotetext{
${ }^{20}$ Il ruolo della famiglia nell'autismo, in clinicalpedagogy.com.
} 
Un ascolto efficace del familiare o del paziente e un'attenzione particolare agli atteggiamenti del corpo, ai comportamenti, ai possibili cambiamenti di umore e alle espressioni del viso, sono gli strumenti di indagine per il caregiver che contribuiscono a realizzare la raccolta delle informazioni necessarie per capire le necessità del familiare e per creare sempre una migliore sintonia.

Proprio per questo la comunicazione deve essere semplice e con una gestualità armoniosa.

Quando il familiare ammalato riesce ad esprimersi e ad essere cosciente è una grande occasione per accompagnarlo durante sua malattia (Parkinson ad esempio).

Se la persona ammalata o disabile viene accudita da un/una badante di nazionalità straniera che non parla bene la lingua italiana, questa persona inizialmente dovrà fare un grande sforzo per capire meglio il contesto in cui vive l'ammalato e le modalità con cui questi si pone rispetto alla sua malattia o al suo problema fisico e mentale. In questi casi entra in gioco la fiducia dell'ammalato nei confronti del familiare, della badante, fiducia che da istintiva nelle prime settimane, deve diventare consapevole (cioè, affido ad un'estranea/o al mio nucleo familiare me stesso e il mio stato di salute nei casi di disabilità o quando la malattia non intacca la lucidità mentale).

\section{La gestione dello stress}

Il caregiver deve essere abile nel gestire la propria emozione, il proprio impegno, sforzandosi nel non considerare al centro della sua attenzione la malattia del familiare (cosa più facile a dirsi se non si è avuto in casa un caso concreto). I momenti di difficoltà dovuti ad un compito molto delicato e inizialmente imprevisto, che implica stanchezza per un impegno così difficile e gravoso, coinvolgono pienamente il caregiver, cosciente della stanchezza dovuta all'impegno di affrontare giornalmente gesti ripetitivi, orari delle medicine e assistenza morale.

Quello che il caregiver deve fare è cercare di rispettarsi, di apprezzarsi per quello che fa ma allo stesso tempo deve cercare di trovare momenti di svago; deve dare massima attenzione al sopraggiungere di possibili sintomi di depressione e nel caso, deve riconoscere nei professionisti del settore la possibilità di aiuto; deve considerare che la conoscenza della patologia del familiare lo aiuta nel rapporto; deve avere la consapevolezza che qualcosa è cambiato e che al suo precedente compito, all'interno del nucleo familiare, se ne è aggiunto un altro: quello di assistere un congiunto. Attraverso questi accorgimenti il caregiver deve affrontare e vivere l'ulteriore nuovo ruolo all'interno del nucleo familiare. Solo attraverso questi accorgimenti e mediante una forte consapevolezza, il caregiver riesce a migliorare il livello di stress che la vita di ogni giorno lo porta a sopportare. La gestione dello stress riguarda molto spesso anche il soggetto assistito, oltre a riguardare i soggetti con handicap che praticano attività motoria o sportiva. Per cercare in questi soggetti di mantenere un buon equilibrio mentale e psicofisico è possibile intervenire consigliando appositi integratori alimentari, mentre per ridurre il danno alle funzioni cellulari è possibile ricorrere a soluzioni tecnologiche. Per migliorare il benessere e le performance dei caregivers e degli atleti portatori di handicap, il supporto tecnologico ${ }^{21}$ potrebbe essere considerato quale ulteriore e possibile elemento per sviluppare l'interesse verso l'attività motoria. Nelle palestre e nei centri sportivi, caregivers e atleti con problemi motori, potrebbero praticare attività fisica, utilizzando strumentazioni e tecnologie in grado di aiutarli a svolgere meglio e con più soddisfazione le loro attività.

Oggi, infatti, esistono strumenti tecnologici che permettono alle proteine attaccate dai radicali liberi e da questi danneggiate, di riprendere le loro funzioni attraverso l'aumento della capacità di riparazione del corpo, aiutando il ripristino della forma tridimensionale della proteina.

\section{Emozioni, stress e rischi nel caregiver}

Quando il caregiver assiste un familiare capace di intendere e di volere, entrambi, in modi differenti, dovrebbero avere la consapevolezza che qualcosa è cambiato rispetto al rapporto familiare precedente. Questo è possibile con una comunicazione adeguata e senza mai sottovalutare l'importanza del contatto fisico quando il proprio familiare non riesce più a comunicare o quando non riesce a fare gesti prima consueti.

\footnotetext{
${ }^{21}$ Per rafforzare il sistema immunitario, aumentare la vitalità e rallentare il processo di invecchiamento, la tecnologia americana brevettata "NanoVi" produce lo stesso segnale biologico di cui il corpo ha bisogno per riparare i danni cellulari causati dai radicali liberi. Il segnale dal dispositivo NanoVi integra quelli che il corpo produce naturalmente.
} 
Chiunque sia coinvolto nel ruolo di caregiver vive una condizione emotiva molto forte. Le emozioni che scaturiscono quando ci si prende cura della persona assistita, in particolare se è un familiare o una persona amata, hanno una intensità tale da generare un forte stress emotivo. Alcune persone subiscono una variazione emotiva talmente forte che li porta ad annullare quasi completamente se stessi per dedicare totalmente le proprie attenzioni ed energie all'assistito.

In quanto esseri umani le emozioni fanno parte della nostra esistenza e hanno un'influenza molto forte sul nostro corpo: possono arrivare a guarirlo ma possono a volte anche danneggiarlo in modo irreparabile.

La letteratura medica riconosce da tempo l'influsso delle emozioni come una delle cause dello sviluppo di patologie o del presentarsi di eventi traumatici per il corpo. ${ }^{22}$

Quando ci si prende cura di altre persone, lo stile di vita del caregiver cambia notevolmente e assume delle connotazioni che non rispecchiano quelle ideali per il mantenimento di un corretto equilibrio. Tale modificazione agisce sia da un punto di vista psicologico che da un punto di vista biologico.

Il carico di stress che si genera si riversa pesantemente sui meccanismi biologici dell'organismo: l'equilibrio tra radicali liberi prodotti e la naturale capacità di contrasto ad essi, viene fortemente compromesso e genera un terreno fertile per l'insorgere di problemi correlati allo stress ossidativo, avviando così processi di invecchiamento precoce.

\section{Prendersi cura di sé per prendersi cura degli altri}

L'importanza dell'attenzione verso sé stessi è un punto fondamentale per chi da assistenza agli altri. Sino ad ora abbiamo visto l'influenza dello stress ossidativo e dei rischi ad esso correlati ai quali ci si può esporre, ma dobbiamo anche tenere ben presente che questo non è l'unico aspetto che deve essere considerato nel valutare i rischi ai quali sono esposti i caregivers.

Esistono infatti anche componenti psicologiche molto forti che entrano in gioco e che possono generare condizioni particolarmente critiche. Per questi motivi è necessario che il caregiver ponga molta attenzione al proprio benessere e alla propria salute, insieme a quella del proprio assistito.

In riferimento agli effetti che le condizioni di stress di coloro che forniscono assistenza possono creare nel corpo, molte ricerche dimostrano come la condizione emotiva è tanto amplificata quanto più è elevato il legame emotivo con la persona da accudire.

In alcuni casi, oltre a subire condizioni psicologiche che possono divenire critiche, il caregiver ha un radicale cambio di stile di vita, che spesso riduce fino ad annullare l'attenzione verso sé stesso. L'insieme di tutti gli stress subiti quasi sempre modifica le abitudini, riduce l'attività fisica e comporta profondi mutamenti con conseguenze sul fisico, sulla mente e sulla psiche.

Questo insieme di condizioni negative si ripercuote in maniera importante sugli equilibri dell'organismo, i quali si alterano con uno sbilanciamento verso la maggiore produzione di radicali liberi, generando una condizione preparatoria per il manifestarsi di problematiche e patologie successive.

Il caregiver deve tenere sempre presente un principio molto importante: prima di assistere una persona è opportuno mettersi nelle migliori condizioni psicofisiche per poter sopportare l'enorme carico cui questa attività sottopone.

\section{Cosa può fare un caregiver per preservare il proprio equilibrio psicofisico}

La condizione altamente stressante che questa realtà genera, deve essere bilanciata dal mantenimento di un corretto stile di vita. È importante mantenere questa condizione per ridurre il rischio di incorrere in conseguenze successive dovute agli squilibri che possono generarsi nel corpo a seguito di queste particolari condizioni.

Come è possibile preservare una buona condizione psicofisica?

\footnotetext{
${ }^{22}$ P. Poprac et al., Targeting Free Radicals in Oxidative Stress-Related Human Diseases, in Trends in pharmacological sciences, 2017, 387, pp. 592-607.
} 
È possibile farlo in molti modi: la prima regola è quella di non dimenticarsi mai di sé stessi, di tenere presente sempre anche le necessità della propria persona. Questo aiuta a mantenere una consapevolezza che permette al caregiver di avere una valutazione oggettiva anche del proprio stato di salute. Inoltre, in quanto il nostro corpo in condizioni particolarmente stressanti utilizza molte risorse di energia che deve produrre, è opportuno curare con attenzione la propria alimentazione, nello stesso modo in cui lo si fa con l'assistito.

L'alimentazione è infatti la principale fonte di reperimento degli elementi necessari per garantire un funzionamento ottimale dell'organismo e per fornire le energie necessarie. Un buon piano alimentare permette di affrontare queste situazioni dolorose e difficili con una maggiore consapevolezza e con maggiori benefici psicofisici, reggendo meglio lo stress subito.

Nelle condizioni di assistenza il caregiver potrebbe facilmente trovarsi con uno squilibrio di nutrienti o minerali, pertanto anche l'utilizzo di integratori alimentari mirati, diventa un supporto molto efficace. Prodotti antiossidanti (per cercare di mantenere più equilibrata possibile l'equilibrio dei radicali liberi), integratori di vitamine e minerali di cui sia nota la carenza, supportano l'organismo per il mantenimento di una buona condizione psicofisica.

Il sonno e il riposo sono elementi fondamentali per la nostra biologia e per regolarne il funzionamento. Durante il sonno infatti il nostro organismo si rigenera in quanto si attuano milioni di processi rigenerativi fondamentali per le cellule che servono a preservare la nostra salute psicofisica. Il caregiver deve mantenere un buono stato di riposo.

Un altro elemento fondamentale è il movimento (l'attività fisica quotidiana) che aiuta, in particolare se viene svolto all'aperto. I benefici del movimento sono conosciuti e in questo caso svolgono anche la funzione di scaricare, attraverso l'attività motoria, buona parte delle tensioni accumulate durante i periodi dedicati alla cura della persona da assistere.

Il caregiver quindi non ha solo il compito di avere cura del suo assistito ma ha anche il compito di non dimenticare mai l'attenzione nei riguardi di sé stesso. È importante che si curi il più possibile, compatibilmente con le esigenze della persona curata. Dimenticarsi di sé, donandosi totalmente agli altri, è una condizione che genera uno stress elevatissimo che ha come conseguenza quella di agire sullo squilibrio di molti processi biologici che sono parte attiva in moltissimi processi di sviluppo di patologie. Per tutti questi motivi esposti la "categoria" dei caregivers è una categoria da salvaguardare per la preziosità del loro compito di assistenza, per il bene del loro assistito e per il bene che ognuno di loro deve volere a se stesso per il fine ultimo che in fondo è il bene dell'altro.

Dopo questa particolare e complessa analisi è superfluo suggerire al Legislatore di migliorare la proposta di legge, di renderla più aderente ad una realtà che solo i soggetti coinvolti riescono a capire le conseguenze. Migliorare la vita e la salute di questa categoria vuol dire migliorare la salute di quei cittadini che svolgono un importante compito al quale un adeguato provvedimento normativo può aiutare a migliorare l'esistenza, dando loro una dignità maggiore rispetto ad un presente senza tutele. 